\title{
Terapêutica da Forma Crônica da Doença de Chagas. É Eficaz o Tratamento Etiológico?
}

\author{
Barbara Maria lanni, Charles Mady \\ São Paulo, SP
}

A doença de Chagas ainda é endêmica na América Latina. Calcula-se que $25 \%$ dos habitantes, cerca de $90 \mathrm{mi}-$ lhões de pessoas, estão expostas ao risco de contrair a doença, havendo 16 milhões de infectados ${ }^{1}$. No Brasil, as estatísticas mostram que cinco a seis milhões de indivíduos estão infectados, havendo 6000 óbitos por ano relacionados à doença ${ }^{2}$.

Esta doença não se expressa sempre da mesma forma. Desde sua descrição por Carlos Chagas, sabemos que, aproximadamente, $60 \%$ das pessoas infectadas estão na forma indeterminada, isto é, têm reações sorológicas positivas para a doença, são assintomáticas, e têm eletrocardiograma (ECG), radiografia de tórax e estudo contrastado de esôfago e cólon normais ${ }^{3,4}$. Vinte e cinco a 35\% dos pacientes têm comprometimento cardíaco, sendo que em apenas $10 \%$ há desenvolvimento de cardiopatia importante 5 .

Como em todas as doenças desencadeadas por agentes infecciosos, foram feitas várias tentativas de tratamento etiológico, com uma série de drogas, com resultados, freqüentemente, desanimadores. Mesmo uma das drogas mais experimentadas, o nifurtimox, foi abandonado pelos efeitos colaterais importantes que apresentava, estando atualmente fora do mercado.

Quando surgiu o benzonidazol, houve nova onda de entusiasmo em relação ao tratamento etiológico, que, em muitos centros, persiste até hoje.

Sabemos que as drogas tripanozomicidas são supressoras de parasitemia e que, para agir no parasita em sua forma intracelular, as doses teriam que atingir níveis tóxicos, não devendo ser utilizadas na clínica ${ }^{6}$. Além disso, por algum tempo o parasita foi relegado a segundo plano na etiopatogenia da doença, já que se acreditava que, após a fase aguda, o processo seguiria um curso imunológico. Hoje, as evidências mostram que o parasita continua presente no organismo do hospedeiro e, provavelmente, é sua presença que mantém as reações imunológicas que, comprovadamente, existem no paciente chagásico ${ }^{7,8}$. Em todas as situações em que as defesas do organismo estão

Instituto do Coração do Hospital das Clínicas - FMUSP

Correspondência: Barbara Maria Ianni - Incor - Av. Dr. Enéas C. Aguiar 44 - 05403 000 - São Paulo, SP

Recebido para publicação em 1/9/97

Aceito em 13/11/97 diminuídas, como durante imunossupressão, há reativação da doença.

Quanto ao uso de tripanozomicidas na fase aguda da doença, não há dúvidas quanto à sua indicação. Porém, quanto à fase crônica, as condutas não são unânimes.

A primeira dificuldade reside no que se poderia utilizar como critério de cura. Tradicionalmente, o xeno foi utilizado, apesar de ser um exame de baixa sensibilidade ${ }^{9}$. Tentando superar isso, são escolhidos, para os estudos, pacientes chamados de alta parasitemia, com três xenodiagnósticos positivos em seguida. Mesmo assim, a variabilidade do teste pode persistir, como demonstrou Cançado ${ }^{10} \mathrm{em}$ seguimento longo de pacientes com xenos repetidos, mostrando que mesmo pacientes com parasitemia moderada podem temporariamente negativá-los sem nenhuma intervenção terapêutica.

Os trabalhos iniciais nos últimos 30 anos foram comparativos entre nifurtimox e benzonidazol. Boainaim mostrou que o benzonidazol era mais efetivo na negativação do xeno que o nifurtimox (93\% x 44\%), havendo ainda efeitos colaterais importantes, como a neurite, dependente da dose, e a dermatite, mais relacionada à sensibilidade à droga ${ }^{11}$.

A superioridade do benzonidazol em relação ao nifurtimox na negativação do xeno foi também comprovada por Ferreira ${ }^{12} \mathrm{em} 100$ pacientes na forma indeterminada e por Levi e col ${ }^{13} \mathrm{em} 49$ pacientes crônicos, sendo 17 na formaindeterminada.

Uma segunda fase da terapêutica específica, relacionada ao aspecto imunológico, foi a inclusão das reações sorológicas nos critérios de cura, como no trabalho de Ferreira ${ }^{14} \mathrm{com} 20$ pacientes na forma indeterminada tratados com benzonidazol, havendo pequena porcentagem de negativação da sorologia. O que se notou, então, foi uma dissociação entre as porcentagens de negativação do xeno (altas) e de negativação das reações sorológicas (baixas ou inexistentes). Esse fato ajudou a aumentar a polêmica sobre o assunto.

Uma terceira fase incluiu grupos controles e aspectos clínicos. Macedo e col, seguindo por sete anos 180 pacientes na forma indeterminada, tratados com nifurtimox ou benzonidazol e 89 tomando placebo, observaram que, em relação a alterações eletrocardiográficas, $6,7 \%$ do grupo tratado e $8,8 \%$ no grupo placebo apresentaram mudanças no ECG, sem diferença estatisticamente significativa ${ }^{15}$. O mesmo não observaram Viotti e col ${ }^{16}$, em estudo com benzo- 
nidazol e seguimento de oito anos de 201 pacientes, 70 tomando placebo; observando diferença estatisticamente significativa entre os grupos, com melhor evolução no grupo tratado quanto às alterações eletrocardiográficas e à mudança de grupo clínico, mais evidente nos pacientes com idade $<50$ anos. Fragata $\mathrm{F}^{\mathrm{o}}{ }^{17}$, seguindo por sete anos $71 \mathrm{pa}-$ cientes tratados com benzonidazol e comparando-os com 49 controles observou também que havia deterioração clínica mais freqüente no grupo controle (14\%) que no grupo tratado (7\%), porém os efeitos colaterais apareceram freqüentemente, sendo que apenas $30 \%$ dos pacientes permaneceram assintomáticos. Em um outro trabalho de observação do uso do benzonidazol com grupo controle, Miranda e col ${ }^{18}$ constataram, após seguimento de até 16 anos, que os pacientes tratados tinham maior porcentagem de estabilização do quadro clínico $(89,5 \%$ x $10,5 \%)$ e que $45 \%$ tiveram efeitos colaterais, que obrigaram a suspensão da medicação em $10 \%$ dos casos, números muito mais promissores que outros trabalhos semelhantes, mesmo usando doses usuais.

Nosso grupo também teve uma experiência com o uso de benzonidazol, em 33 pacientes na forma indeterminada, sendo 18 no grupo controle. Dois pacientes tiveram alterações eletrocardiográficas após nove anos de seguimento, ambos do grupo que tomou a droga e 1/3 do grupo tratado apresentou efeitos colaterais importantes, o suficiente para a suspensão do tratamento ${ }^{19}$. Este é um aspecto nem sempre abordado com clareza nos trabalhos publicados.

Na faixa etária pediátrica, o trabalho de Andrade e $\mathrm{col}^{20}$ mostrou boa tolerância ao benzonidazol (menos de 5\% de efeitos colaterais entre 64 pacientes tratados) com seroconversão de 63,7\% com a droga, além de diminuição de títulos após o tratamento e de menor porcentagem de aparecimento de alterações eletrocardiográficas durante três anos de seguimento. Como se pode observar, a literatura não é concordante quanto aos resultados terapêuticos com essa droga.

Outras drogas já foram testadas recentemente, dentre elas o alopurinol. Meirovich e $\mathrm{col}^{21}$, na Argentina, compararam nifurtimox, benzonidazol, alopurinol em dois esquemas terapêuticos e placebo e concluíram que a eficácia era seme- lhante na maior dose utilizada da droga $(7 \mathrm{a} 10 \mathrm{mg} / \mathrm{kg} /$ dia por 60 dias) em relação ao nifurtimox e ao benzonidazol, sem aparecimento de efeitos colaterais importantes, que existiam em até $50 \%$ dos pacientes usando as outras drogas, freqüentemente, obrigando a suspensão do tratamento ${ }^{21}$. Porém esses dados não puderam ser reproduzidos no Brasil, talvez pela diferença de cepa ${ }^{22}$. Boainaim e $\mathrm{col}^{23}$ observaram, em 11 pacientes, que apenas um negativou o xeno após o tratamento.

Também recentemente foi experimentado o ketoconazol, com resultados desanimadores, como demonstrou Brener e $\mathrm{col}^{24} \mathrm{em}$ oito pacientes. O mesmo aconteceu com o itraconazol, utilizado por Fragata $\mathrm{F}^{\circ}$ e $\mathrm{col}^{25} \mathrm{em} 12$ pacientes, com repositivação do xeno em 100\% dos casos após dois anos de seguimento.

No Brasil, uma normatização de 1996 do Ministério da Saúde recomenda o tratamento na fase crônica para as crianças e os adultos jovens na forma indeterminada, sendo que, nos outros casos, a conduta deveria ser individualizada ${ }^{26}$. Como tivemos oportunidade de recentemente demonstrar, os pacientes na forma indeterminada têm boa evolução, desenvolvendo alterações eletrocardiográficas em pequena porcentagem dos casos, sem correspondente alteração de fração de ejeção de ventrículo esquerdo, independente de qualquer intervenção ${ }^{27}$. E, conforme comentamos, os resultados terapêuticos são absolutamente discordantes, não nos gerando uma segurança baseada em dados objetivos da literatura para sua utilização. Será que devemos mesmo tratar esse grupo de pacientes?

Pelo exposto, continuamos no mesmo impasse: tratar ou não tratar? Como podemos tomar a decisão de usar uma droga potencialmente tóxica, de manejo não tão fácil, num grande número de pacientes?

Continuamos sem resposta para essa questões. Além do esforço da pesquisa básica em relação a novas drogas, nós, clínicos, temos a responsabilidade de definir melhores critérios de cura ou pelo menos controle da doença, e de acompanhar a longo prazo os pacientes chagásicos, antes de ficarmos completamente convencidos de ter tomado a decisão acertada.

\section{Referências}

1. World Health Organization - Control of Chagas' disease. Report a WHO Expert Committee. Geneva. WHO Technical Report Series 1991; 811: 1-95.

2. Dias JCP, Dias E- Doença de Chagas. In: Brasil. Ministério da Saúde - SUCAM. Doença de Chagas: texto de apoio. Brasília, 1989: 13-20.

3. Macedo V - Inquérito eletrocardiográfico nacional para doença de Chagas. Rev Soc Bras Med Trop 1993; 26: 12-3.

4. Primeira Reunião de Pesquisa Aplicada em Doença de Chagas. Validade do conceito de forma indeterminada. Rev Soc Bras Med Trop 1985; 18: 46.

5. Dias JCP - Profilaxia e impacto médico-social da doença de Chagas na região mineira do polígono das secas. Rev Goiana Med 1982; 28: 97-102.

6. Boainain E, Rassi A - Terapêutica etiológica da doença de Chagas. Arq Bras Cardiol 1979; 32: 395-9.

7. Higuchi ML-O parasita e a patogenia da forma crônica da doença de Chagas. Arq Bras Cardiol 1995; 64: 251-4.
8. Cunha Neto E, Kalil J - Auto-imunidade na doença de Chagas. Rev Soc Cardiol ESP 1994; 4: 92-100

9. Bronfen E, Alvarenga NJ - O xenodiagnóstico e os critérios para avaliar o nível de parasitemia do paciente chagásico crônico. Rev Soc Bras Med Trop 1991; 24: $37-42$.

10. Cançado JR - Tratamento específico. In: Cançado JR, Chuster M - Cardiopatia Chagásica. Belo Horizonte. Fundação Carlos Chagas 1985; 33: 327-55.

11. Boainain $\mathrm{E}$ - Tratamento etiológico da doença de Chagas na fase crônica. Rev Goiana Med 1979; 25: 1-60.

12. Ferreira HO - Tratamento da forma indeterminada da doença de Chagas com nifurtimox e benzonidazol. Rev Soc Bras Med Trop 1990; 23: 209-11.

13. Levi GC, Lobo IMF, Kallás EG, Amato Neto V - Etiological drug treatment of human infection by Trypanosoma cruzi. Rev Inst Med Trop São Paulo 1996; 38: $35-8$. 
14. Ferreira HO - Ensaio terapêutico-clínico com o benzonidazol na doença de Chagas. Rev Inst Med Trop São Paulo 1976; 18: 357-64.

15. MacedoV, Silveira CAN, Maués WR - Influência da terapêutica específica na evolução da forma indeterminada da doença de Chagas. Rev Soc Bras Med Trop 1984; 17(supl): 34

16. Viotti R., Vigliano C, Armenti H, Segura E-Treatment of chronic Chagas' disease with benznidazole: clinical and serologic evolution of patients with long term follow-up. Am Heart J 1994; 127: 151-62.

17. Fragata $\mathrm{F}^{\circ} \mathrm{AA}$ - Tratamento etiológico da doença de Chagas. In: Sousa AGMR, Mansur AJ, eds - Cardiologia. Soc Cardiol ESP 1996; 2: 598-603.

18. Miranda L, Miranda L, Campos G et al - História natural da forma crônica da doença de Chagas x tratamento específico. Rev Centro-Oeste Cardiol 1994; 1: 25 9.

19. Ianni BM, Arteaga E, Mady C et al - Uso do benzonidazol em chagásicos na forma indeterminada: resultados a longo prazo. Arq Bras Cardiol 1993; 61(supl II): 130.

20. Andrade ALSS, Zicker F, Oliveira RM et al - Randomised trial of efficacy of benznidazole in treatment of early Trypanosoma cruzi infection. Lancet 1996; 348: 1407-13
21. Meirovich CI, Montrull HL, Gallerano RH, Sosa RR - Alopurinol en el tratamiento de la enfermedad de Chagas cronica. Arq Bras Cardiol 1985; 45 217-3.

22. Amato Neto V - Tratamento específico da doença de Chagas. Rev Hosp Clín Fac Med SP 1980; 35: 27-34.

23. Boainain E, Pereira VL, Levy AMA, Barboza CM, Ramos MC - Tentativa do tratamento específico da doença de Chagas na fase crônica com alopurinol riboside. Mem Inst Oswaldo Cruz. Rio de Janeiro 1990(supl I); 85: 101.

24. BrenerZ, Cançado JR, Galvão LMC et al - An experimental and clinical assay with ketoconazole in the treatment of Chagas' disease. Mem Inst Oswaldo Cruz. Rio de Janeiro 1993; 88: 149-53.

25. Fragata $\mathrm{F}^{\circ} \mathrm{AA}$, Boainain $\mathrm{E}$, Maifrino LBM et al - Itraconazol in treatment of chronic Chagas'disease. Mem Inst Oswaldo Cruz 1993; 88(suppl): 243.

26. Amato Neto V (relator) - Reunião sobre tratamento etiológico da doença de Chagas. Ministério da Saúde. Fundação Nacional de Saúde. Departamento de Operações. Gerência Técnica da Doença de Chagas, 1996.

27. Ianni BM - Forma indeterminada da doença de Chagas: avaliação evolutiva de parâmetros clínicos, eletrocardiográficos e ecocardiográficos (tesedoutoramento). São Paulo. USP, 1995; 102p. 